\title{
Epidermoid Cyst of the Fourth Ventricle: A Case Report
}

\author{
Sushil Kumar ${ }^{1}$ Sandeep Sharma ${ }^{1} \quad$ Rajneesh Misra ${ }^{1} \quad$ Kundan Kumar $^{1}$ \\ ${ }^{1}$ Department of Neurosurgery, St Stephen's Hospital Tis Hazari, \\ Delhi, India

\begin{abstract}
Address for correspondence Sandeep Sharma, MBBS, MS (Surgery), DNB Neurosurgery, Department of Neurosurgery, St Stephen's Hospital Tis
\end{abstract} \\ Hazari, Delhi 110054, India (e-mail: sandy_mamc05@yahoo.co.in).
}

Indian J Neurosurg 2019;8:191-192
Abstract
Keywords
- epidermoid cyst
- fourth ventricle
- magnetic resonance
imaging
Epidermoid cysts of the fourth ventricle are slow-growing benign rare lesions. We report a case of fourth ventricle epidermoid in a young lady with short duration of symptoms.

\section{Introduction}

Epidermoid cysts account for $0.2-1.8 \%$ of all intracranial tumors. ${ }^{1}$ They have been described by various names-tumor perlee, pearl tumor, and cholesteatoma. ${ }^{2}$ Usually they occur throughout the neuraxis, most commonly being at cerebellopontine angle and location in the fourth ventricle is uncommon and being symptomatic for a short duration, hence it is being reported.

\section{Case Report}

A 30-year-old woman presented with history of occipital headache, difficulty in walking for 1.5 months, and off and on episodes of vomiting and blurring of vision for 1 month. Headache had increased in severity during the last 10 days prior to admission. On examination the patient was conscious. There were bilateral cerebellar signs. Magnetic resonance imaging (MRI) performed outside revealed an extraaxial large cystic lesion centered within and expanding the fourth ventricle. On T1WI, the lesion appeared hypointense and hyperintense on T2WI. There was restriction on DWI and lesion did not enhance on gadolinium administration (-Fig. 1). Hydrocephalus was present. MRI was suggestive of typical intraventricular epidermoid cyst. Right ventriculoperitoneal shunt was followed by suboccipital craniectomy. Pearly white tumor filling the fourth ventricle could be easily separated from the floor of the ventricle and it was removed completely. The patient had uneventful postoperative course.

received

December 31, 2018

accepted after revision

March 26, 2019

published online

November 4, 2019
Histopathology confirmed the diagnosis of epidermoid. On telephonic interview the patient is reported to be asymptomatic for 5 years and 7 months and MRI done in Assam had showed no recurrence.

\section{Discussion}

Epidermoid cysts are benign slow-growing congenital tumors and develop from the inclusion of ectodermal cells during the closure of neural tube between the third and fifth week of intrauterine life $^{3}$ and most authors consider epidermoid to be congenital malformations rather than true neoplasms. ${ }^{4,5}$ Although congenital in origin, they manifest between the third and fifth decade. ${ }^{3}$ Most patients have long clinical symptomatology running into years. ${ }^{6}$ Only two cases have been reported with symptomology of 4 and 6 weeks, respectively. ${ }^{2,7}$

Rarely these lesions may be acquired following trauma and lumbar puncture; however, it is uncommon, secondary to trauma in the brain. Fourth ventricle epidermoid may present with headache, vertigo, difficulty in walking, vomiting, and mental impairment. ${ }^{3}$ Our patient had symptoms and signs of raised intracranial pressure and cerebellar dysfunction. They grow and insinuate within cerebrospinal fluid spaces surrounding and encasing adjacent vessels and nerves. On computed tomography the typical appearance is that of a nonenhancing and hypoattenuating extraaxial mass. Calcification has been reported in $10-25 \%$ cases. MRI is the best modality for evaluating these lesions. Characteristically they are isointense relative to cerebrospinal fluid on T1WI and 


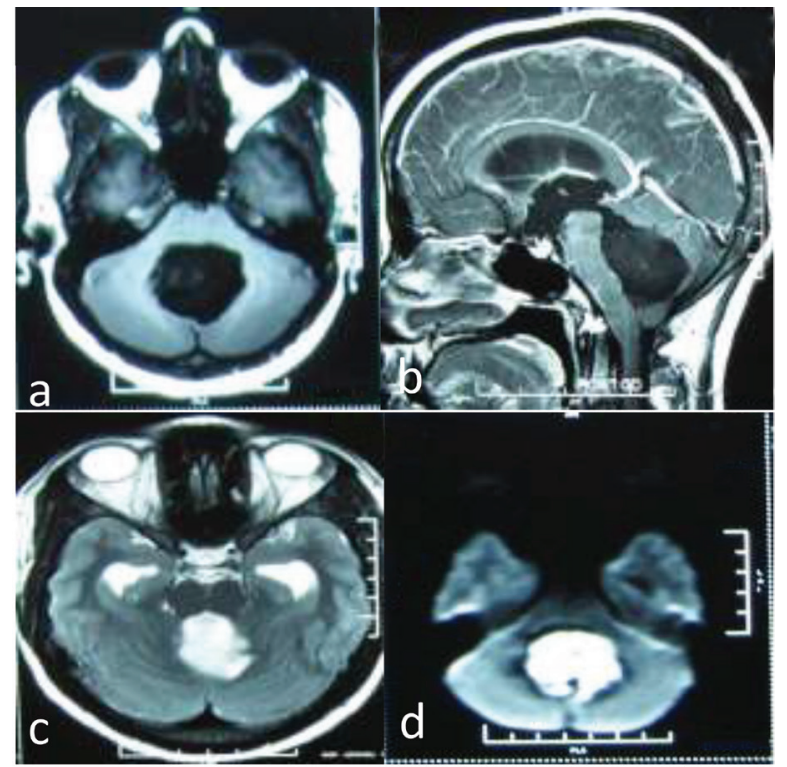

Fig. 1 (a) Large hypointense lesion on T1WI in the fourth ventricle. (b) T1WI with gadolinium not showing any enhancement. (c) T2WI sequence showing hyperintense lesion. (d) DWI showing restriction.

T2WI. At times they may show high signal intensity on T1WI and low signal intensity on T2WI due to high protein content and are referred to as white epidermoids. ${ }^{8}$ Hydrocephalus is not commonly seen with intracranial epidermoids but was present in our patient necessitating ventriculoperitoneal shunt.

Differential diagnosis of fourth ventricular epidermoid includes arachnoid cyst, dermoid cyst, cystic neoplasm, and hydatid cyst. Arachnoid cyst lacks diffusion restriction. ${ }^{2}$ Dermoids show hyperintense signal on T1-weighted images. ${ }^{2}$ Cystic medulloblastoma and ependymoma usually have heterogeneous appearance due to hemorrhage and necrosis and exhibit heterogenous contrast enhancement. ${ }^{2}$

Epidermoid should be extirpated totally to avoid recurrence, but, not at the risk of increasing deficit by leaving adherent portion to vital structures. ${ }^{1}$ Spilling into subarachnoid space should be avoided to prevent chemical meningitis. ${ }^{6}$ If it occurs it is treated by corticosteroids administration through repeated lumbar puncture. ${ }^{9}$ Malignant transformation, though rare, should be suspected if rapid progression of neurological symptoms occur and tumor enhancement is there. ${ }^{10}$

\section{Conflicts of Interest}

None declared.

\section{Referneces}

1. Nagasawa D, Yew A, Safaee M, et al. Clinical characteristics and diagnostic imaging of epidermoid tumors. J Clin Neurosci 2011;18(9):1158-1162

2. Nassar SI, Haddad FS, Abdo A. Epidermoid tumors of the fourth ventricle. Surg Neurol 1995;43(3):246-251

3. Hossini A, Lakhdar F, Gana R, et al. [Epidermoid cyst of the cisterna magna and the fourth ventricle: Report of four cases]. Neurochirurgie 2012;58(6):358-363

4. Niikawa S, Hara A, Zhang W, Sakai N, Yamada H, Shimokawa K. Proliferative assessment of craniopharyngioma and epidermoid by nucleolar organizer region staining. Childs Nerv Syst 1992;8(8):453-456

5. Russel DS, Rubinstein LJ. Dermoid and epidermoid cysts. In: Russel DS, Rubinstein LJ, eds. Pathology of Tumours of the Nervous System. 5th ed. London, United Kingdom: Edward Arnold; 1989:690-702

6. Tancredi A, Fiume D, Gazzeri G. Epidermoid cysts of the fourth ventricle: very long follow up in 9 cases and review of the literature. Acta Neurochir (Wien) 2003;145(10):905-910, discussion 910-911

7. Scott M. Epidermoid tumour (tumor perlée, cholesteatoma) of the fourth ventricle: case report and review of literature. J Neurol Neurosurg Psychiatry 1974;37(12):1329-1332

8. Osborn AG, Preece MT. Intracranial cysts: radiologic-pathologic correlation and imaging approach. Radiology 2006;239(3): 650-664

9. Meng L, Yuguang L, Shugan Z, Xingang L, Chengyuan W. Intraventricular epidermoids. J Clin Neurosci 2006;13(4):428-430

10. Michael LM II, Moss T, Madhu T, Coakham HB. Malignant transformation of posterior fossa epidermoid cyst. Br J Neurosurg 2005;19(6):505-510 